\title{
The effect of Korean traditional alternative medicine products on thyroid function level in post- total thyroidectomy patients: A pilot study
}

\author{
Joon-Hyop Lee, Yoo Seung Chung, Young Don Lee \\ Thyroid and Endocrine Surgery Section, Department of Surgery, Gachon University Gil Medical Center, Incheon, Korea
}

Purpose: Although many Koreans consume traditional alternative remedial products to alleviate symptoms of fatigue, there have been no studies on their effect on thyroid function levels in thyroidectomized patients on levothyroxine replacement. The aim of this study was to assess the effect of Korean traditional alternative remedies on thyroid function levels in post total thyroidectomy patients.

Methods: A retrospective review of medical records from a single tertiary referral center was performed on patients who received total thyroidectomy from 2010 to 2015 and became euthyroid postoperatively. Gender, age, body mass index, preoperative thyroid function test results, interval to first hypothyroidism occurrence, recurrence of hypothyroidism, number and types of alternative remedy were evaluated.

Results: Postoperative hypothyroidism occurred among 174 out of 917 patients (18.9\%) and 100 (57.5\%) of them had a history of alternative medication usage. The first episode of hypothyroidism occurred at median of 38.5 months after operation in the 100 patients. Six of the 100 patients received an increased dosage of levothyroxine due to severe hypothyroidism but the rest received the same dose and were requested not to consume alternative products. All patients recovered to euthyroid status afterwards. Thirty-three patients subsequently experienced recurrence even after being restricted from consuming alternative remedial products. Apart from thyroiditis on pathology reports $(P=0.001)$, there were no variables significantly related to the recurrent hypothyroidism.

Conclusion: This pilot study demonstrated the possible role of consumption patterns of traditional alternative remedial products in thyroidectomized patients under hormone supplement in restoring euthyroid status without levothyroxine increase.

Keywords: Complementary therapies, Herbal drug interactions, Thyroidectomy, Thyroid function tests, Hypothyroidism

\section{INTRODUCTION}

Despite apparently adequate thyroid hormone replacement, many patients experience persistent hypothyroid symptoms after receiving total thyroidectomy $[1,2]$. Post-treatment fatigue is one of the most commonly reported complaints among thyroid cancer sur-

Received: May 30, 2017 Accepted: Jun 23, 2017

Correspondence to: Young Don Lee

Thyroid and Endocrine Surgery Section, Department of Surgery, Gachon University School of Medicine, 21 Namdong-daero 774beon-gil,

Namdong-gu, Incheon 21565, Korea

Tel: +82-32-460-3244, Fax: +82-32-460-3247

E-mail: peacemk@gilhospital.com

*This study was conducted at the Gachon University Gil Medical Center, Gachon University School of Medicine.

Copyright $@$ Korean Society of Surgical Oncology

This is an Open Access article distributed under the terms of the Creative Commons Attribution Non-Commercial License (http://creativecommons.org/licenses/by-nc/4.0) which permits unrestricted non-commercial use, distribution, and reproduction in any medium, provided the original work is properly cited. vivors, and statistically significant worse levels of fatigue compared with the general or healthy population were reported in those survivors [3]. It is well believed in Korea that traditional herbal medicine or supplementary food products can alleviate symptoms of fatigue, and thus many thyroid cancer survivors who received total thyroidectomy rely on such treatments $[4,5]$.

According to the clinical practice guidelines for hypothyroidism issued in 2012 by the American association of clinical endocrinologists and the American thyroid association, agents such as multivitamins containing ferrous sulfate or calcium carbonate, grapefruit juice, soy, high fiber diet, etc. [6] are reported to interfere with the absorption of oral thyroid hormone supplements (levothyroxine). Levothyroxine is mostly absorbed at the proximal small bowel within four to five hours after intake and the products mentioned above interfere with the absorption if consumed within this time period. Some postulate that certain traditional alternative remedies and food products could have similar effects on hormone absorption [7]. There has been no study, however, on the ef- 
fect of Korean traditional alternative remedial products on thyroid hormone levels of thyroidectomized patients on levothyroxine supplementation.

In the present study, we conducted a pilot research project to assess the effect of alternative medicine and food products on thyroid function levels in post total thyroidectomy patients.

\section{METHODS}

A retrospective review of medical records from a single tertiary referral center was performed after the study protocol concerning human materials was approved by the Institutional Review Board (IRB no., GBIRB 2017-104). This study was conducted at the Gachon University Gil Medical Center, Gachon University School of Medicine.

\section{Patient follow-up}

All patients who received total thyroidectomy regardless of their diagnosis were administered oral levothyroxine and instructed not to take any traditional alternative remedies or supplementary products. They were followed up 2 weeks, 3 months after operation and biannually henceforth. During each outpatient session, thyroid stimulating hormone (TSH; range, $0.4-4.87 \mathrm{IU} / \mathrm{mL}$ ) and free T4 (0.8-1.78 ng/dL) levels were checked to assess the effectiveness of the hormone replacement therapy. If TSH levels were above normal but below $30 \mathrm{IU} / \mathrm{mL}$, patients were asked if they took any alternative remedies, re-educated if they had and were followed up at the next planned outpatient session without altering the dose of levothyroxine. If the TSH level was above $30 \mathrm{IU} / \mathrm{mL}$, levothyroxine dosage was increased and patients were followed up one month later to be checked for thyroid function level recovery.

\section{Patient selection}

Among the patients who received total thyroidectomy between 2010 and 2015 by a single surgeon and returned to euthyroid status shortly afterwards, those who took traditional alternative remedial products and, simultaneously, whose TSH level was above normal were included in the analysis. Exclusion criteria included pregnancy during spells of hypothyroidism, incorrect administration methods of levothyroxine tablets, and free T4 levels below normal range.

\section{Data analysis}

All patients' gender, age at operation (years), body mass index (BMI, $\mathrm{kg} / \mathrm{m}^{2}$ ), preoperative TSH $(\mathrm{IU} / \mathrm{mL}$ ), preoperative free T4 $(\mathrm{ng} / \mathrm{dL})$, type of thyroiditis reported on pathology review, concurrent medication administration, interval from operation to first hypothyroidism spell (months), recurrence of hypothyroidism, interval between first and recurred hypothyroidism (months), types and number of alternative products, and total follow-up period (months) were analyzed. The clinical characteristics of those who had recurrent hypothyroidism even after the first occurrence were compared to those who did not experience repeated incidence of hypothyroidism after the first event.

\section{Statistical analysis}

Continuous data were presented as mean \pm standard deviation or median (minimum-maximum value) unless stated otherwise. Clinical parameters were compared using Student's t-test or Fisher's exact test. P-values $<0.05$ were considered statistically significant. Statistical analyses were performed with SPSS ver. 20.0 software package (SPSS Inc., Chicago, IL, USA).

\section{RESULTS}

Among the 917 patients who received total thyroidectomy 174 (18.9\%) experienced episodes of postoperative hypothyroidism. One hundred (57.5\%) among them satisfied both the inclusion and exclusion criteria and were followed up for a median time period of 66 months. The baseline demographic and clinical characteristics of these 100 patients are summarized in Table 1. The male to female ratio was 1:4 and the median age at operation was 48 years. The mean preoperative TSH and free T4 levels were $2.4 \mathrm{IU} /$

Table 1. Patient characteristics

\begin{tabular}{lc}
\hline Characteristic & $\mathrm{n}=100$ \\
\hline Sex & \\
$\quad$ Male & 20 \\
Female & 80 \\
Age at operation (yr) & $48(17-75)$ \\
Body mass index (kg/m²) & $23.6 \pm 3.6$ \\
Preoperative TSH (IU/mL) & $2.4 \pm 7.0$ \\
Preoperative free T4 (ng/dL) & $1.3 \pm 0.2$ \\
Thyroiditis & \\
$\quad$ None & 67 \\
Lymphocytic & 23 \\
Hashimoto & 10 \\
Concurrent medication administration & 41 \\
Interval to first hypothyroidism (mo) & $38.5(3-123)$ \\
Recurred hypothyroidism & \\
$\quad$ No & 67 \\
Yes & 33 \\
Interval to recurred hypothyroidism (mo) & $23(11-44)$ \\
Number of alternative products & $1.6 \pm 1.0$ \\
Total follow-up period (mo) & $66(14-1159)$ \\
\hline
\end{tabular}

$\mathrm{TSH}$, thyroid stimulating hormone. 
$\mathrm{mL}$ and $1.3 \mathrm{ng} / \mathrm{dL}$, respectively, while 41 patients were on other medications due to hypertension ( $\mathrm{n}=28)$, diabetes mellitus $(\mathrm{n}=7)$, gastritis $(\mathrm{n}=5)$, arthritis $(\mathrm{n}=4)$, asthma $(\mathrm{n}=3)$, depression $(\mathrm{n}=1)$, osteoporosis $(\mathrm{n}=1)$, and Parkinson's disease $(\mathrm{n}=1)$.

The first episode of hypothyroidism occurred in 33 patients at a median of 38.5 months period after operation. The mean TSH and free T4 value was $14.19 \mathrm{UI} / \mathrm{mL}$ and $1.21 \mathrm{ng} / \mathrm{dL}$, respectively. During history taking, all patients revealed that they had been taking at least one type of alternative remedy either to alleviate symptoms of fatigue or just as a supplement for recuperating (Table 2). The types of product taken did have statistically significant influence on the episodes of recurrent hypothyroidism. Six (17.6\%) patients whose TSH level was higher than $30 \mathrm{IU} / \mathrm{mL}$ received an increased dosage of levothyroxine while the rest of the patients were only re-educated to not take any alternative remedies without levothyroxine dose adjustments. Within the next outpatient session or two, the patients' thyroid function recovered and all patients became euthyroid.

However, 33 patients subsequently experienced recurrent episodes of hypothyroidism even after being told not to take alternative remedial products. All of them resorted to another type of alternative remedy after being recommended to stop the alternative one. The median interval between the first and recurrent episodes

Table 2. Types of alternative remedy products

\begin{tabular}{lcc}
\hline Type of products & No. of patients & $\begin{array}{c}\text { Difference in recurrent } \\
\text { hypothyroidism episodes } \\
\text { (P-value) }\end{array}$ \\
\hline Red ginseng (Panax ginseng) & 48 & 0.407 \\
Mugwort (Artemisia species) & 18 & 0.701 \\
Herbal tea products & 46 & 0.204 \\
Various sap products & 32 & 0.054 \\
Mushroom products & 16 & 0.640 \\
\hline
\end{tabular}

of hypothyroidism was 23 months and all 33 patients disclosed their continuous usage of alternative remedy. The statistical analysis between patients with and without recurrent hypothyroidism revealed that the presence of thyroiditis on the permanent pathology report was the only significant factor between the two groups $(\mathrm{P}=0.001)$. Other parameters such as gender $(\mathrm{P}=0.349)$, age $(\mathrm{P}=0.848)$, BMI $(\mathrm{P}=0.372)$, preoperative TSH $(\mathrm{P}=0.331)$, preoperative free T4 $(\mathrm{P}=0.847)$, TSH after first adjustment $(\mathrm{P}=0.669)$, free T4 after first adjustment $(\mathrm{P}=0.403)$, concurrent medication due to other medical conditions ( $\mathrm{P}=0.752)$, and types and number of alternative remedies taken $(\mathrm{P}=0.342)$ did not reveal statistical significance (Table 3 ). There were no significant differences in the number of alternative remedies taken between the thyroiditis and non-thyroiditis groups $(\mathrm{P}=0.080)$.

\section{DISCUSSION}

The present research was a pilot study on the effect of traditional Korean remedial products on post-total thyroidectomy thyroid function level. In this study we described the various types of supplementary products consumed by post-patients and the patterns in which hypothyroidism occurs and recurs in these patients. We discovered that 174 out of 917 patients (18.9\%) experienced episodes of postoperative hypothyroidism and that 100 (57.5\%) of them had a history of alternative medication usage. We also found out that in $67 \%$ of cases post-thyroidectomy hypothyroidism were prevented from recurring without increasing the dosage of levothyroxine but by merely warning patients about the effects that remedial medicine could have on the absorption of levothyroxine.

There are many causes of hypothyroidism for post-thyroidectomy patients despite adequate hormone replacement. Endocrine, autoimmune, hematological, metabolic, and lifestyle issues are reported as possible causes of persistent hypothyroid symptoms in

Table 3. Factors related to recurrent episodes of hypothyroidism

\begin{tabular}{lccc}
\hline Medication for & $\begin{array}{c}\text { Recurred hypothyroidism } \\
\text { group }(\mathrm{n}=33)\end{array}$ & $\begin{array}{c}\text { Non- recurred hypothyroidism } \\
\text { group }(\mathrm{n}=67)\end{array}$ & $\begin{array}{c}\text { P-value } \\
\text { Sex (male) }\end{array}$ \\
Age $(\mathrm{yr})$ & $48.8 \pm 15.1)$ & $48.3 \pm 10.3$ & 0.349 \\
Body mass index $\left(\mathrm{kg} / \mathrm{m}^{2}\right)$ & $23.2 \pm 3.6$ & $23.8 \pm 3.3$ & 0.848 \\
Preoperative TSH & $1.5 \pm 1.1$ & $3.0 \pm 9.0$ & 0.372 \\
Preoperative free T4 & $1.3 \pm 0.3$ & $1.3 \pm 0.2$ & 0.331 \\
TSH post-adjustment & $1.0 \pm 1.0$ & $1.0 \pm 1.1$ & 0.847 \\
Free T4 post-adjustment & $1.5 \pm 0.3$ & $1.6 \pm 0.3$ & 0.669 \\
Thyroiditis & $13(39.3)$ & $6(9.0)$ & 0.403 \\
No. of alterative medication & $1.7 \pm 1.0$ & $1.5 \pm 0.9$ & 0.001 \\
Concurrent medication & $2(6.1)$ & $3(4.5)$ & 0.342 \\
\hline
\end{tabular}

TSH, thyroid stimulating hormone. 
patients on synthroid supplementation [6,8]. In Korea, however, many people rely on traditional remedies, which they believe interact more naturally with human metabolism. Several guidelines state certain nutritional products and drugs as agents that disrupt the absorption of levothyroxine but few studies mention the effect that Korean traditional remedies have on its absorption. This is a factor that should be taken into consideration when dealing with Korean patients.

Amidst the lack of pertinent studies, ours was the first to assess the effect of traditional Korean remedies on post-thyroidectomy thyroid function. This pilot study was meaningful in that it demonstrated 67 out of 100 postoperative hypothyroid patients taking some type of traditional remedy retain euthyroid status with education alone and without levothyroxine dose increase. Reducing the excessive intake of thyroid hormones can help prevent adverse effects such as atrial fibrillation or osteoporosis [8].

But as this was a pilot study, it has many limitations. First of all, it was a retrospective study and there were no set protocols in the education, follow-up and description of the subjects. Secondly, because we relied on the patients' memory to recall what type and amount of remedial products they consumed the data was not thoroughly reliable. Moreover, the quantity of such products was impossible to assess and therefore we could only conduct a qualitative assessment on the remedy consumption. Finally, the total number of participants was not large. All these elements led to a lack of statistical significance when we compared the different factors between the recurred and non-recurred hypothyroidism groups. Existence of thyroiditis, which was the only statistically significant factor, can be a risk factor for post-lobectomy hypothyroidism [9] and therefore needs further evaluation to gain clinical meaning. In order to overcome such limitations, a prospective study with a larger number of participants with a predefined protocol to ascertain the type, amount, and interval of the alternative remedy and prescribed medication, and also the change in severity of symptoms such as fatigue and weight gain is necessary, which we are planning to conduct in the future.

This pilot study revealed the wide usage of traditional alternative remedial products and the importance of considering their consumption patterns before increasing the levothyroxine dose in post-total thyroidectomy Korean patients. Although the evidence is not sound enough to be incorporated into clinical management guidelines as a high-level recommendation at the moment, further prospectively designed randomized control trials with quantitative analyses may provide sounder evidence in the future.

\section{CONFLICT OF INTEREST}

No potential conflict of interest relevant to this article was reported.

\section{REFERENCES}

1. Saravanan P, Chau WF, Roberts N, Vedhara K, Greenwood R, Dayan CM. Psychological well-being in patients on 'adequate' doses of 1-thyroxine: results of a large, controlled community-based questionnaire study. Clin Endocrinol (Oxf) 2002;57:577-85.

2. Wekking EM, Appelhof BC, Fliers E, Schene AH, Huyser J, Tijssen JG, et al. Cognitive functioning and well-being in euthyroid patients on thyroxine replacement therapy for primary hypothyroidism. Eur J Endocrinol 2005;153:747-53.

3. Sawka AM, Naeem A, Jones J, Lowe J, Segal P, Goguen J, et al. Persistent posttreatment fatigue in thyroid cancer survivors: a scoping review. Endocrinol Metab Clin North Am 2014;43:475-94.

4. Kaneko H, Nakanishi K. Proof of the mysterious efficacy of ginseng: basic and clinical trials: clinical effects of medical ginseng, Korean red ginseng: specifically, its anti-stress action for prevention of disease. J Pharmacol Sci 2004;95:158-62.

5. Chen R, Moriya J, Yamakawa J, Takahashi T, Kanda T. Traditional chinese medicine for chronic fatigue syndrome. Evid Based Complement Alternat Med 2010;7:3-10.

6. Garber JR, Cobin RH, Gharib H, Hennessey JV, Klein I, Mechanick JI, et al. Clinical practice guidelines for hypothyroidism in adults: cosponsored by the American Association of Clinical Endocrinologists and the American Thyroid Association. Thyroid 2012;22:1200-35.

7. Miller LG. Herbal medicinals: selected clinical considerations focusing on known or potential drug-herb interactions. Arch Intern Med 1998;158:2200-11.

8. Okosieme O, Gilbert J, Abraham P, Boelaert K, Dayan C, Gurnell $\mathrm{M}$, et al. Management of primary hypothyroidism: statement by the British Thyroid Association Executive Committee. Clin Endocrinol (Oxf) 2016;84:799-808.

9. Shin HS, Ko JW, Kim JS, Moon DJ. Risk factors for hypothyroidism after thyroid lobectomy with papillary thyroid crcinoma according to existence of thyroiditis. Korean J Endocr Surg 2011; 11:90-6. 